\title{
Imaging Findings in Patients with Granulomatous Mastitis
}

\author{
Pelin Seher Oztekin, ${ }^{1,}$ Gamze Durhan, ${ }^{1}$ Pinar Nercis Kosar, ${ }^{1}$ Serap Erel, ${ }^{2}$ and Sema Hucumenoglu ${ }^{3}$ \\ ${ }^{1}$ Radiology Department, Ankara Training and Research Hospital, Ankara, Turkey \\ ${ }^{2} 4$ th Surgery Department, Ankara Training and Research Hospital, Ankara, Turkey \\ ${ }^{3}$ Pathology Department, Ankara Training and Research Hospital, Ankara, Turkey \\ "Corresponding author: Pelin Seher Oztekin, Radiology Department, Ankara Training and Research Hospital, Ankara, Turkey. E-mail: pelinoztekintr@yahoo.com
}

Received 2015 October 18; Revised 2016 February 11; Accepted 2016 March 02.

\begin{abstract}
Background: Granulomatous mastitis (GM) is a rare inflammatory breast disease that may mimic the clinical characteristics and radiologic imaging findings of breast carcinoma. Considering the importance of making a correct diagnosis, careful radiologic evaluations and recognition of imaging features are necessary.

Objectives: The aim of this study was to review the radiological findings and diagnostic value of the imaging in GM.

Patients and Methods: This retrospective study involved a total of 29 patients who were diagnosed with GM between 2009 and 2013 and who underwent mammography (MG) and/or ultrasound (US) examination in addition to magnetic resonance imaging (MRI) before diagnosis.

Results: Among 14 patients over 35 years of age who underwent MG imaging, focal asymmetric, ill-defined nodular, or diffusely increased densities were detected in nine (64.3\%), two (14.3\%), and one (7.1\%) subjects, respectively, while there were no pathological findings in two (14.3\%) patients. In the overall group of 29 patients, US showed heterogeneous hypoechoic lesions with tubular extensions in 16 (55.2\%), well-demarcated heterogeneous hypoechoic lesions in eight (27.6\%), parenchymal heterogeneous appearance in three (10.3\%), and a heterogeneous hypoechoic lesion with irregular margins in one (3.4\%), with another (3.4\%) patient having normal US findings. MRI findings included lesions consistent with solitary or multiple separate or confluent abscesses with marked peripheral ring enhancement in 25 (86.2\%) patients, accompanied by intensity changes suggesting edematous inflammation in the peripheral parenchyma, as well as non-mass-like heterogeneous segmental and regional contrast enhancement. Four (13.8\%) patients had non-mass-like segmental and regional contrast enhancement only. A histopathological diagnosis of GM was established in all patients with biopsy.

Conclusion: GM presents with a wide range of conventional radiological findings, hampering the diagnosis. In patients with inconclusive conventional findings, MRI may assist in the differential diagnosis and assessment of the extent of disease. However, a definitive diagnosis and relevant treatment require histopathological confirmation.
\end{abstract}

Keywords: Granulomatous Mastitis, Breast, Mammography, Ultrasonography

\section{Background}

Granulomatous mastitis (GM) is an uncommon benign inflammatory condition of the breast. Idiopathic GM, originally described by Kessler and Wolloch in 1972 (1), represents a sub-group of GM with unknown etiology. The remaining cases of GM are associated with infectious conditions, such as fungal infections, actinomycosis, histoplasmosis, brucellosis, and tuberculosis in particular, as well as with other conditions, such as Wegener's granulomatosis and sarcoidosis (2). The real incidence of GM is unknown, with only a few hundred cases reported in the literature(3). Clinically, radiologically, and even cytologically, it can be confused with malignancy, requiring histopathological examination for a definitive diagnosis $(4,5)$. Conventional radiological findings are non-specific and exhibit wide variation. Magnetic resonance imaging (MRI) has emerged as an important diagnostic tool, providing certain advantages over other imaging modalities in the differential diagnosis of breast conditions.

\section{Objectives}

In this study, conventional radiological and MRI findings were evaluated in patients with GM, with an emphasis on the value of the imaging modality and the diagnostic role of radiology in GM.

\section{Patients and Methods}

This retrospective study involved a total of 29 GM patients between 20 and 69 years of age (mean \pm SD: $35.14 \pm$ 9.9) who were diagnosed with GM between 2009 and 2013 
in our clinic. The study protocol was approved by the Institutional Ethics Committee (project number: 0545).

Mammography(MG) examinations were performed in all patients over the age of 35 years (14/29) in standard craniocaudal and mediolateral-oblique projections (Lorad MIV, Hologic). In the remaining 15 patients (under age 35), MG was not performed.

All patients underwent ultrasound (US) and MRI examinations, and the results were documented. Highresolution US images (Xario SSA-660A, Toshiba) were obtained by a linear-array transducer with a center frequency of 7.5 MHz. MRI indications included exclusion of inflammatory cancer in treatment-resistant cases, further assessment in patients with inconclusive MG and/or sonography results, and determination of the extent of disease. MRI was performed after conventional examinations in all patients in a manner that would not result in any treatment delay. MRI examinations were performed with a 1.5-T whole-body imaging system (Signa Excite, GE Healthcare, Milwaukee, WI, USA). The patients were scanned in the prone position with the breast suspended in a fourchannel breast coil. MR images were obtained in the transverse and sagittal planes with fat suppression. Precontrast transverse acquisitions were performed using a T1-weighted fast spin-echo sequence and transverse T2weighted fast spin echo short-tau inversion recovery(STIR) imaging, and pre-contrast sagittal acquisitions were performed using a T2-weighted fast spin echo sequence with fat suppression. Sagittal pre and post-contrast dynamic imaging was performed using a 3D multi-phase fast gradient echo pulse sequence called VIBRANT (flip angle $10^{\circ}$; minimum echo time $2.4 \mathrm{msec}$; maximum echo time 14.0 msec; section thickness $3 \mathrm{~mm}$ with no intersection gap; field of view $20 \mathrm{~cm}$; matrix size $256 \times 256$; NEX 1; one signal acquired; imaging time, 1 minute for each phase). Additionally, transverse post-contrast T1-weighted images were acquired using the fast spoiled gradient-recalledecho sequence in the same manner used to acquire the pre-contrast images, without a change in the patient's position. Subtraction images were done. The patients were given a bolus intravenous injection of gadolinium contrast ( $0.2 \mathrm{mmol} / \mathrm{kg}$ body weight) with a power injector. Both the morphological features and the kinetic characteristics of the lesions were examined. All MR images were reviewed on high-resolution PACS monitors (General Electric Medical Systems).

For a final differential diagnosis, biopsy was recommended in all cases, especially for treatment planning. Twenty-six patients underwent core biopsy, while three had excisional biopsy according to the surgeon's preference. Core biopsy was performed under US guidance using a $14-G$ needle. Due to inconsistent radiological and core biopsy results in four patients, additional excisional biopsies were required. Among all 29 patients, seven required surgical excision of lesions.

A diagnosis of tuberculosis-associated GM was made in 10 patients, and cat-scratch disease-associated GM was diagnosed in one. No causative factors could be determined for the remaining 18 patients.

\subsection{Statistical Methods}

SPSS ver. 15 (SPSS Inc., Chicago, Illinois, USA) and Medcalc statistical software (Belgium) packages were used for statistical analysis. This study consisted of only GM patients and descriptions of their imaging features. Therefore, a percent calculation was performed. As the only statistical analysis, the chi-square $\left(\chi^{2}\right)$ test was used to compare the ratios of the BI-RADS categories between the conventional methods and MRI. A value of $\mathrm{P}<0.05$ was accepted as statistically significant.

\section{Results}

\subsection{Clinical Findings}

The majority $(26 / 29,90 \%)$ of the patients were of reproductive age and none had a history of autoimmune disease, sarcoidosis, or systemic tuberculosis. Two patients had a family history of breast cancer. The symptoms had been present for 15 days to 4 months before a diagnosis was made, and included breast pain (26/29, 90\%), palpable breast mass (23/29, 79\%), and erythema and inflammation (9/29, 31\%). Three (10.3\%) patients had sinus tracts and one (3.4\%) had nipple retraction. The condition was unilateral in all cases, with 20 on the right side (Table 1).

Table 1. Clinical Characteristics of the Patients

\begin{tabular}{|lc|}
\hline Symptom & No. $(\%)$ \\
\hline Breast pain & $26(90)$ \\
\hline Erythema & $9(31)$ \\
\hline Palpable lump & $23(79)$ \\
\hline Sinus tract & $3(10.3)$ \\
\hline Nipple retraction & $1(3.4)$ \\
\hline Axillary lymphadenopathy & $13(45)$ \\
\hline
\end{tabular}

\subsection{Mammography Findings}

Nine of the 14 (64.3\%) patients who underwent MG had dense and heterogeneous dense parenchymal breast patterns. Nine (64.3\%) patients had focal asymmetric densities, two (14.3\%) had ill-defined nodular densities, one (7.1\%) 
had diffuse increased density, and two (14.3\%) had no pathological findings with dense patterns. No pathological calcifications were observed.

\subsection{Ultrasonography Findings}

Sixteen (55.2\%) patients had ill-defined lesions with tubular extensions, eight (27.6\%) had well-demarcated lesions with posterior acoustic enhancement, three (10.3\%) had parenchymal edema-heterogeneity, one (3.4\%) had a mass lesion with irregular borders, and one (3.4\%) had normal results. All lesions exhibited heterogeneous hypoechogenicity. Three (10.3\%) had fistula tracts. Twelve (41.4\%) patients had axillary lymph nodes with mild or moderate enlargement, echogenic hila, and a symmetric or asymmetric thick cortex. One patient (3.4\%) had markedly enlarged lymph nodes with fatty hila, which could not be clearly visualized. Color Doppler US findings were available for 16 patients, and increased arterial and venous vascularization was evident at the lesion level in all cases. Table 2 summarizes the mammography, US, and color Doppler US findings of the $29 \mathrm{GM}$ patients.

Table 2. Mammographic and Ultrasonographic Findings of Patients

\begin{tabular}{|c|c|}
\hline Findings & No. (\%) \\
\hline \multicolumn{2}{|l|}{ Mammography $(n=14)$} \\
\hline Normal & $2(14.3)$ \\
\hline Ill-defined nodular density & $2(14.3)$ \\
\hline Focal asymmetrically increased density & $9(64.3)$ \\
\hline Diffuse increased density & $1(7.1)$ \\
\hline Skin thickening & $3(21.4)$ \\
\hline \multicolumn{2}{|l|}{ Ultrasonography $(n=29)$} \\
\hline Normal & $1(3.4)$ \\
\hline Parenchymal heterogeneity & $3(10.3)$ \\
\hline Het. hypoechoic ill-defined lesion with tubular extensions & $16(55.2)$ \\
\hline Het. hypoechoic lesion with well-defined border & $8(27.6)$ \\
\hline Het. hypoechoic lesion with irregular border & $1(3.4)$ \\
\hline Fistulae & $3(10.3)$ \\
\hline Skin thickening & $5(17.2)$ \\
\hline Unilaterally enlarged axillary lymph nodes & $13(44.8)$ \\
\hline \multicolumn{2}{|l|}{ Color Doppler sonography $(n=16)$} \\
\hline Increased arterial and venous vascularization & $16(100)$ \\
\hline
\end{tabular}

Conventional methods (US/US + MG) could identify 16 out of $29(55.2 \%)$ cases with lesions of probably benign (BIRADS 3) and inflammatory origin. However, these methods could not distinguish the remaining 13 (44.8\%) cases from malignancy; 12 (41.4\%) patients were categorized as having a suspicious abnormality (BI-RADS 4) and one (3.4\%) patient's lesion was highly suggestive of malignancy (BIRADS 5).

\subsection{MRI Findings}

Twenty-five of the 29 (86.2\%) participants had one or more separate or confluent lesions consistent with abscess formation, with regular or irregular borders and marked circumferential contrast enhancement following IV gadolinium injection. These lesions were hyperintense on T2-weighted images (T2WI) and hypointense on T1WI, with variable signal intensities depending on the protein content of the fluid. At the same level of these lesions or adjacent to them were areas with heterogeneous signal changes, without well-defined borders or mass effect, as well as segmental/regional heterogeneous non-mass-like contrast enhancement. The size of the MRI lesions ranged from $5-6 \mathrm{~mm}$ to $5 \mathrm{~cm}$. In four patients (13.8\%), no lesions were observed other than parenchymal heterogeneity and non-mass-like segmental contrast enhancement. Kinetic analyses of non-mass-like contrast-enhanced areas, lesions, and lesion walls showed type 1 kinetic curves in 24 (82.7\%) patients at all levels, type 2 kinetic curves in the lesion walls in four (13.8\%) patients, and type 3 kinetic curves in the lesion walls and non-mass-like contrast-enhanced areas in one $(3.4 \%)$ patient. The patient with type 3 kinetic curves had a positive family history. MRI showed the presence of lesions within only one quadrant in 13 (44.8\%) patients, while multiple quadrants were involved in 16 (55.2\%). Twelve patients had peripheral involvement, two had retroareolar involvement, and seven had both peripheral and retroareolar involvement. Fistula tracts were present in three patients, and 16 (55.2\%) patients had mildly or moderately enlarged axillary lymph nodes with symmetric or asymmetric thick cortex structures on MRI. A single (3.4\%) patient had enlarged lymph nodes without fatty hila. Table 3 summarizes the MRI findings.

On the MRI examinations of the 29 cases, 24 (82.7\%) were deemed probably benign (BI-RADS 3 ) and inflammatory in nature, four (13.8\%) were considered suspicious for malignancy (BI-RADS 4), and one (3.4\%) was considered highly suggestive of malignancy (BI-RADS 5).

Eleven cases interpreted as "suspicious" with conventional methods were considered probably benign based on MRI findings, while two cases assessed as "suspicious" on MRI were considered probably benign based on conventional methods. These two patients had clinicalradiological mismatches and the only MRI finding was non-mass-like segmental contrast enhancement. One patient with markedly enlarged lymph nodes without fatty hila was categorized based on conventional imaging and 
Table 3. MRI Findings of Patients

\begin{tabular}{|c|c|}
\hline MRI Findings & No. (\%) \\
\hline Focal lesion & $25(86.2)$ \\
\hline Solitary lesion with well-defined borders & $11(44)$ \\
\hline Multiple small lesions ( $<1 \mathrm{~cm}$ ) with well-defined borders & $5(20)$ \\
\hline Confluent lesions with irregular margins & $8(32)$ \\
\hline \multicolumn{2}{|l|}{ Lesion signal intensity } \\
\hline Hypointense on T1WI, hyperintense on T2WI & $16(64)$ \\
\hline Intermediate on T1WI, het. hyperintense on $\mathrm{T} 2 \mathrm{WI}$ & $4(16)$ \\
\hline Hypointense on T1WI, het. hyperintense on T2WI & $4(16)$ \\
\hline Het. hypointense on T1WI, and het. hyperintense on T2WI & $1(4)$ \\
\hline \multicolumn{2}{|l|}{ Lesion enhancement } \\
\hline Peripheral ring enhancement & $25(100)$ \\
\hline Parenchymal het. intensity changes & $29(100)$ \\
\hline Non-mass-like enhancement & $4(13.8)$ \\
\hline \multicolumn{2}{|l|}{ Type of enhancement curve } \\
\hline Type 1 & $24(82.7)$ \\
\hline Type 2 & $4(13.8)$ \\
\hline Type 3 & $1(3.4)$ \\
\hline \multicolumn{2}{|l|}{ Other findings } \\
\hline Fistulae & $3(10.3)$ \\
\hline Skin thickening & $12(41.8)$ \\
\hline Unilaterally enlarged axillary lymph nodes & $17(58.6)$ \\
\hline
\end{tabular}

Abbreviation: Het, Heterogeneous; WI, weighted imaging.

MRI as BI-RADS 5 and BI-RADS 4, respectively, and the reverse was true in another patient with a family history. The other cases of conventional and MRI findings were assessed as the same BI-RADS category (Table 4). Compared with conventional methods (55.17\%), the rate of the BI-RADS 3 classification was significantly higher based on $\operatorname{MRI}(82.75 \%$; $=0.006)$.

Figures 1 - 3 show examples of imaging findings from the study participants.

\subsection{Treatment and Follow-Up}

Most of the patients were on antibiotic therapy for findings of breast abscesses at the time of their referral to the radiology clinic. All of the patients consulted a general surgeon for a treatment plan. Before the treatment plan, all of the patients were evaluated for possible respiratory system illnesses (tuberculosis, sarcoidosis, etc.) with chest radiography and/or thoracic computerized tomography (CT). Tuberculin sensitivity tests were also performed on all patients. Biopsy and histopathological as-
Table 4. BI-RADS Assessments of Diagnostic Methods

\begin{tabular}{|c|c|c|c|}
\hline Patient & Age & US/US+MG & MRI \\
\hline 1 & 29 & BI-RADS 4 & BI-RADS 3 \\
\hline 2 & 35 & BI-RADS 4 & BI-RADS 3 \\
\hline 3 & 39 & BI-RADS 3 & BI-RADS 4 \\
\hline 4 & 41 & BI-RADS 3 & BI-RADS 3 \\
\hline 5 & 42 & BI-RADS 3 & BI-RADS 4 \\
\hline 6 & 34 & BI-RADS 3 & BI-RADS 3 \\
\hline 7 & 34 & BI-RADS 4 & BI-RADS 3 \\
\hline 8 & 30 & BI-RADS 4 & BI-RADS 3 \\
\hline 9 & 25 & BI-RADS 3 & BI-RADS 3 \\
\hline 10 & 35 & BI-RADS 3 & BI-RADS 3 \\
\hline 11 & 41 & BI-RADS 3 & BI-RADS 3 \\
\hline 12 & 34 & BI-RADS 3 & BI-RADS 3 \\
\hline 13 & 45 & BI-RADS 4 & BI-RADS 3 \\
\hline 14 & 41 & BI-RADS 5 & BI-RADS 3 \\
\hline 15 & 69 & BI-RADS 5 & BI-RADS 4 \\
\hline 16 & 35 & BI-RADS 4 & BI-RADS 3 \\
\hline 17 & 24 & BI-RADS 3 & BI-RADS 3 \\
\hline 18 & 45 & BI-RADS 3 & BI-RADS 3 \\
\hline 19 & 40 & BI-RADS 4 & BI-RADS 5 \\
\hline 20 & 34 & BI-RADS 3 & BI-RADS 3 \\
\hline 21 & 39 & BI-RADS 3 & BI-RADS 3 \\
\hline 22 & 29 & BI-RADS 4 & BI-RADS 3 \\
\hline 23 & 34 & BI-RADS 3 & BI-RADS 3 \\
\hline 24 & 23 & BI-RADS 3 & BI-RADS 3 \\
\hline 25 & 24 & BI-RADS 3 & BI-RADS 3 \\
\hline 26 & 48 & BI-RADS 4 & BI-RADS 4 \\
\hline 27 & 24 & BI-RADS 3 & BI-RADS 3 \\
\hline 28 & 20 & BI-RADS 4 & BI-RADS 3 \\
\hline 29 & 26 & BI-RADS 4 & BI-RADS 3 \\
\hline
\end{tabular}

Abbreviations: BI-RADS, Breast Imaging-Reporting and Data System; MG, mammography; MRI, magnetic resonance imaging; US, ultrasound.

sessments were performed in all cases, especially for treatment planning (Figure 4). Ten patients were diagnosed with tuberculosis and received antituberculous treatment according to advice of the chest disease specialist. Three of the remaining 19 patients underwent complete excision, and the remainder (16/29) received low-dose steroid therapy $(0.5 \mathrm{mg} / \mathrm{kg} /$ day) for 4 - 8 weeks. After the medical treatment, all of the patients were followed every three months for two years, then every six months for two years, then yearly. There were no recurrences requiring either medi- 

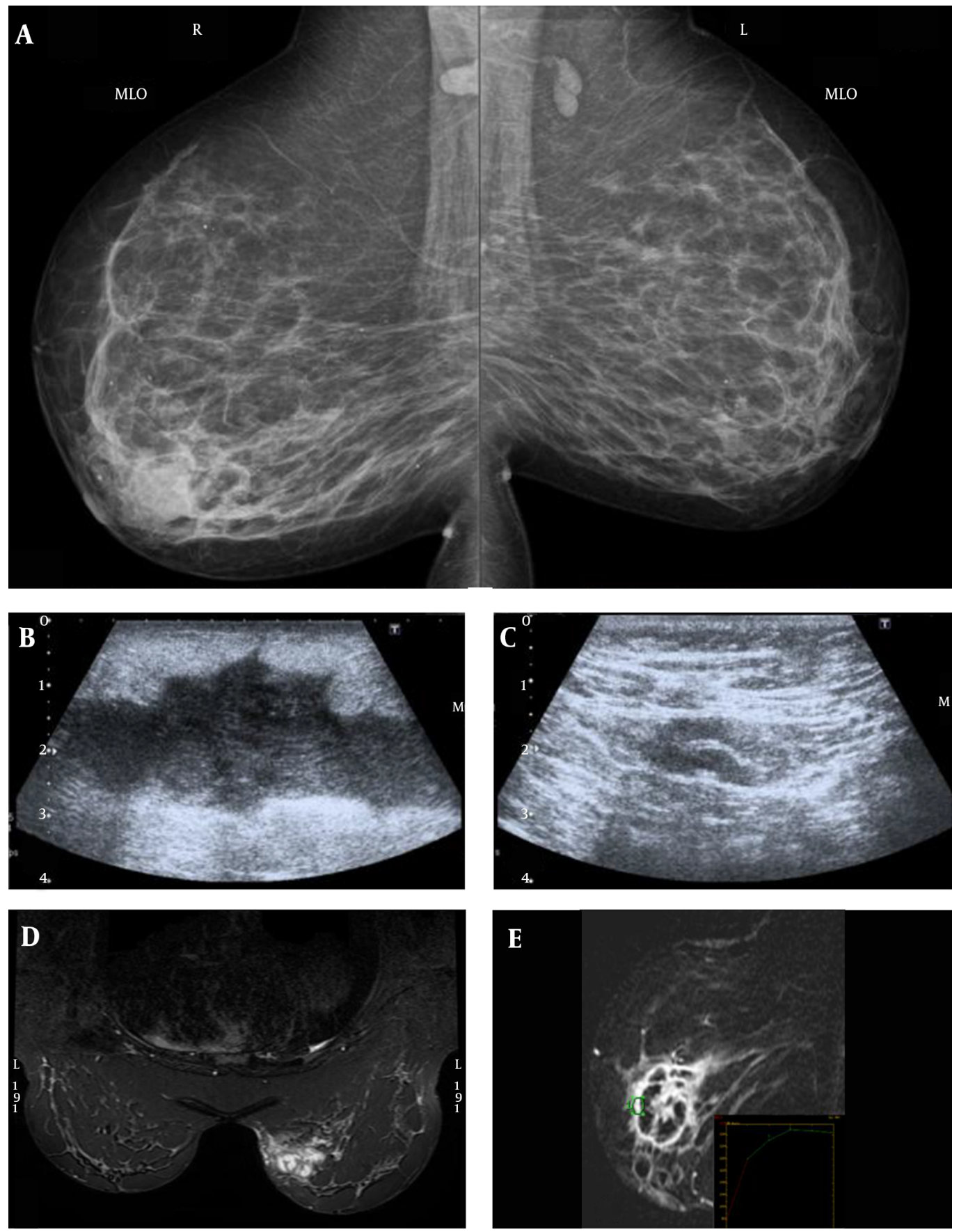

Figure 1. A 35-year old patient (patient no. 2), who presented with a palpable right breast mass. A, Bilateral mediolateral-oblique mammography showed a nodular density surrounded by peripheral fibroglandular tissue. B, Ultrasound showed a heterogeneous hypoechoic lesion with tubular extensions. C, Axillary examination showed moderately enlarged lymph node with thickened cortex. D, STIR axial and E, T1-weighted fat-suppressed post-contrast subtraction sagittal MR images showed a mass lesion with peripheral ring enhancement and irregular borders consistent with abscess formation in the same patient. Type 1 kinetic curve of the lesion wall is seen (E).

cal or surgical therapy in our study group after 39 (15 - 73) months of follow-up. A complete response was observed after therapy; however, this does not ensure that no lesions are present histopathologically. 

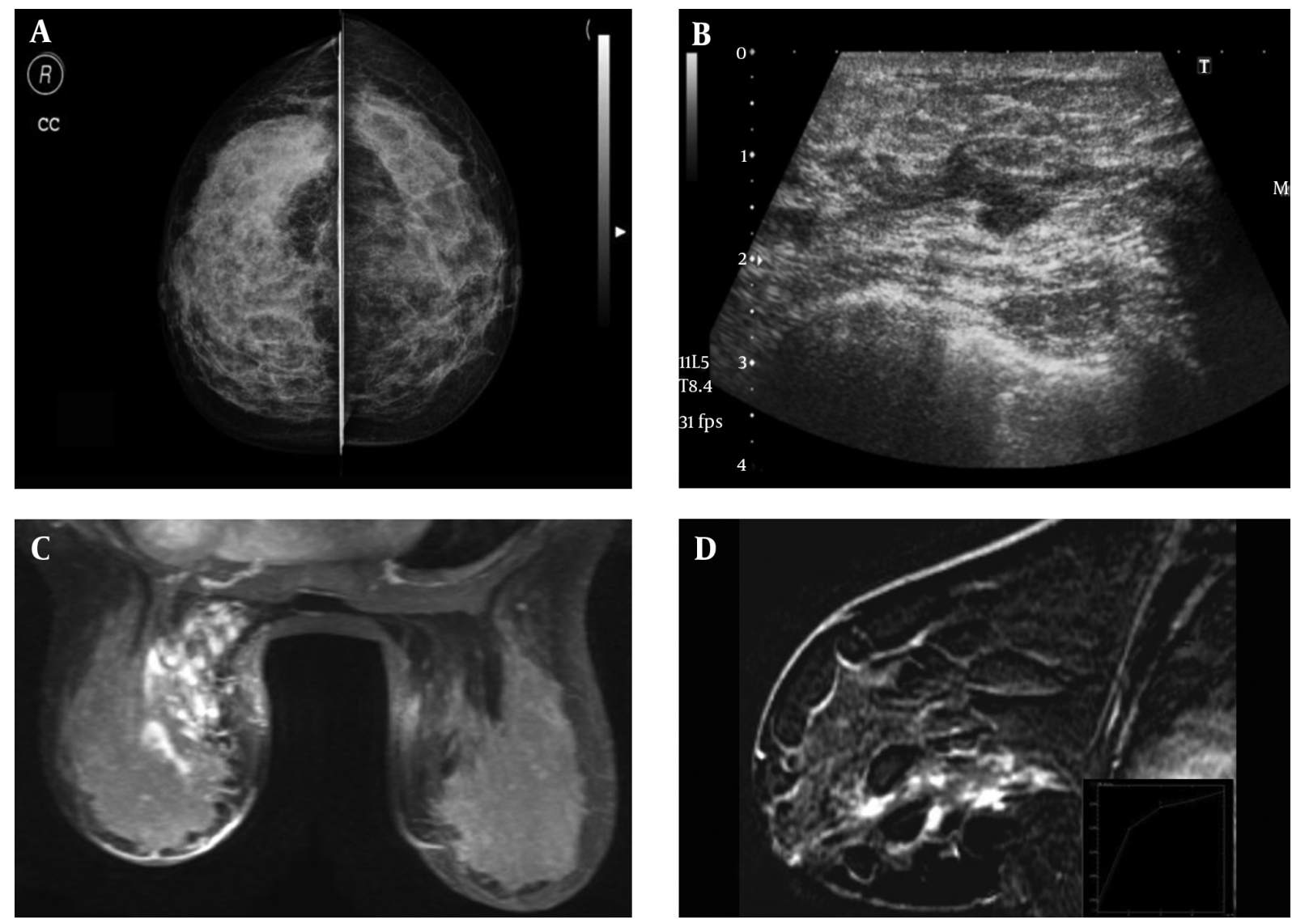

Figure 2. A 42-year-old patient who presented with breast pain (patient no. 5). A, Bilateral craniocaudal mammography showed asymmetric increased density, more prominent in the inner quadrant of the left breast. B, Ultrasound showed a hypoechoic heterogeneous ill-defined lesion, with tubular extensions. C, Post-contrast fat-suppressed maximum intensity projection reformatted axial and D, T1-weighted fat-suppressed post-contrast subtraction sagittal MR images showed non-mass-like segmental contrast enhancement. Type 1 kinetic curves at the level of contrast enhancement is seen (D).

\section{Discussion}

GM is an uncommon inflammatory condition of the breast characterized by granuloma and abscess formation (2), generally affecting young women of reproductive age, mostly during the five years following childbirth (2-8). Until now, the largest patient series reported in the literature was that of Yildiz et al. (8), which involved 30 patients with a diagnosis of idiopathic GM. Thus, to our knowledge, our study represents the second-largest patient series in the literature with an evaluation of radiologic findings of GM.

The absence of complaints or clinical signs suggestive of inflammation despite the presence of a palpable mass in the majority of GM patients, and enlarged axillary lymph nodes in a certain proportion of these subjects, may lead to a suspicion of breast cancer. In addition, GM may not be readily differentiated from breast cancer radiologically (1-18). It generally involves one breast, although bilateral involvement has also been reported $(2,5,7,10)$. In our study, all of our cases had unilateral GM with a predominance of peripheral involvement, and the proportion of patients with retroareolar involvement was higher than that reported previously $(6,11,12)$.

Several studies have reported on MG and US findings in GM patients, and a general consensus exists as to the conventional radiological signs of GM. MG generally shows non-specific signs, the most common of which is focal asymmetric increased density, ill-defined borders, and mass effect, or irregular masses with ill-demarcated borders $(3,8,9,11-15)$. MG has low sensitivity for this condition, particularly when considering the younger population that is affected, as the abovementioned findings may not be detected in women with dense breast tissue $(3,6,13$, 16). The MG findings of the current study are in line with previous reports, and the lesions could not be detected in two of our patients with dense breast tissue.

Since US is commonly used for the initial assessment 

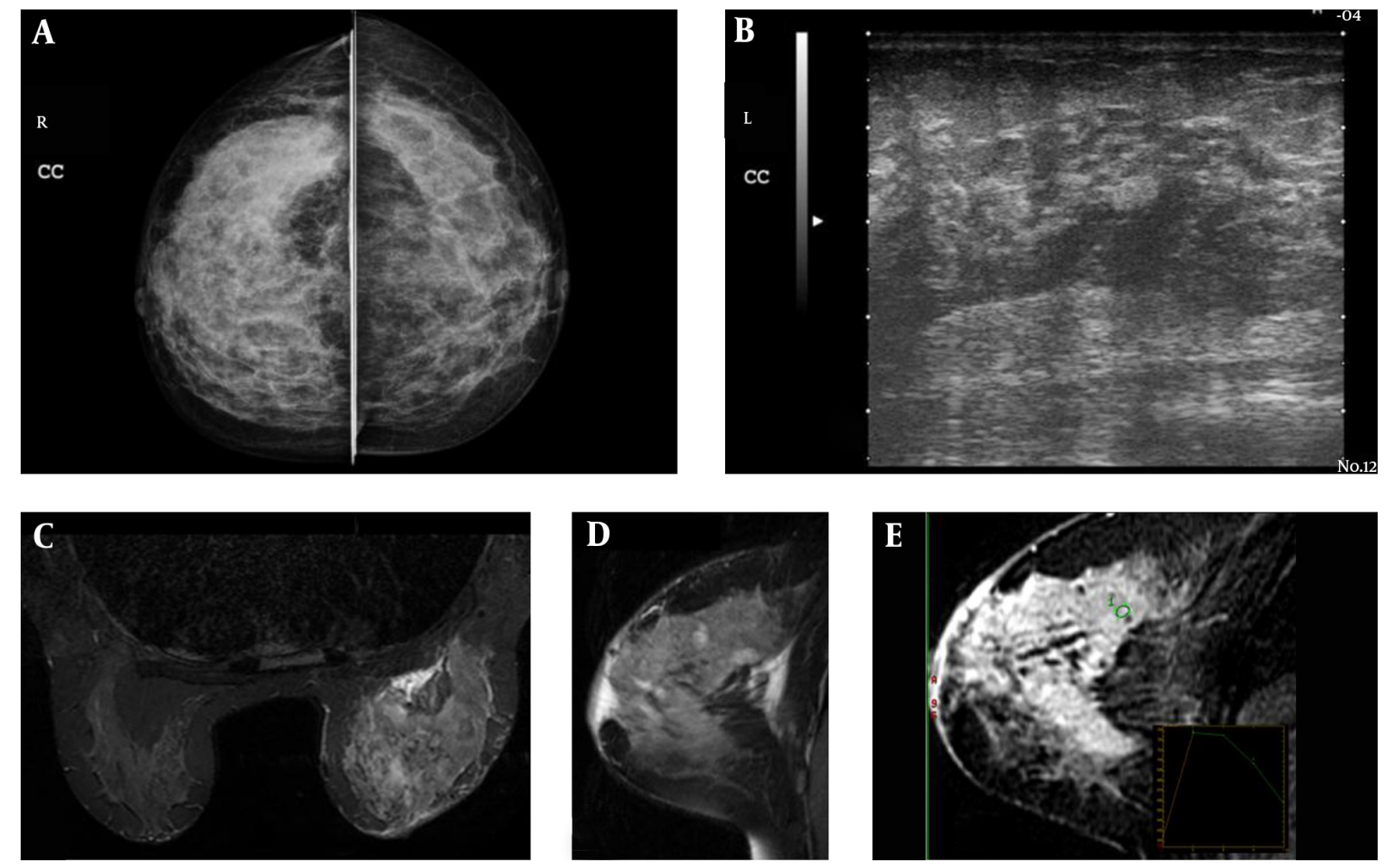

Figure 3. A 40-year-old patient who presented with a palpable breast lesion (patient no. 19). A, Bilateral craniocaudal mammography showed asymmetric increased density, more prominent in the right lateral quadrant of the right breast. B, Ultrasound showed heterogeneous hypoechoic ill-defined lesions with tubular extensions. C, STIR axial, D, T2-weighted fat-suppressed sagittal and E, T1-weighted fat-suppressed post-contrast subtraction sagittal MR images showed parenchymal heterogeneous intensity changes, non-mass-like regional contrast enhancement, and lesions less than $1 \mathrm{~cm}$ in diameter with peripheral contrast enhancement, consistent with micro-abscess formation. Type 3 kinetic curves adjacent to the lesions are seen.

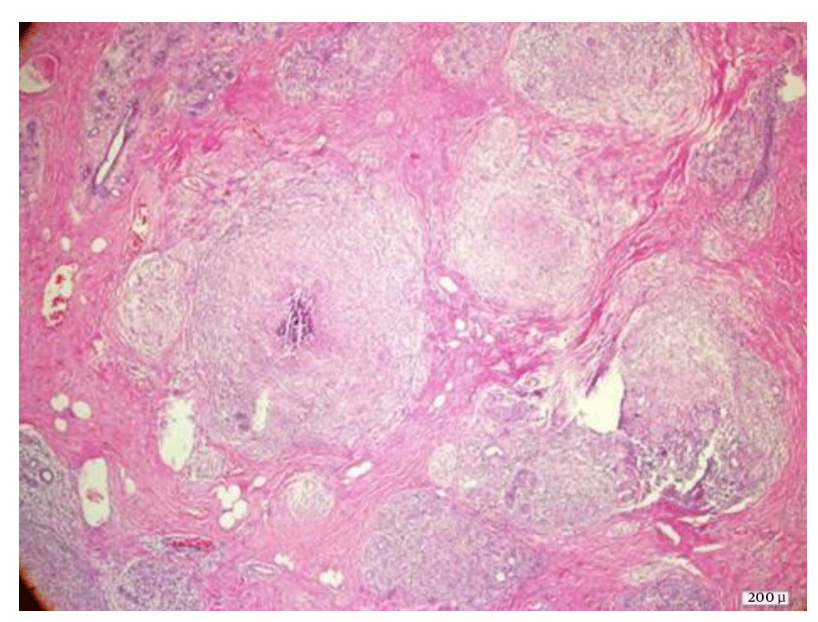

Figure 4. Granulomatous structures characterized by granulomatous reaction $(\mathrm{H} \& \mathrm{E} \times 200)$

of young females with palpable breast lesions, most GM patients undergo US examinations prior to diagnosis. The most common US findings in these patients are heterogeneous hypoechoic ill-defined lesions with tubular extensions, as in our study $(3,6,11,13,15)$. However, other findings, such as hypoechoic lesions with regular or irregular contours, posterior acoustic enhancement or shadowing, parenchymal heterogeneity, and distortions may also be seen, in addition to skin thickening and diffuse parenchymal edema (13). One specific advantage of US is its ability to facilitate aspiration in cases of suspected fluid collection. However, it should be borne in mind that cytology alone may not be adequate to exclude underlying malignancy or to diagnose GM, necessitating a confirmatory histopathological diagnosis (3). In addition, a normal US examination cannot exclude a diagnosis of GM, as demonstrated by one of our cases with asymmetric densities on MG that were not detected by US.

MRI is an imaging modality with high sensitivity and specificity for breast lesions, allowing the detection and assessment of the extent of inflammatory breast conditions. The MRI characteristics of GM have been reported in only a few cases in the literature, and data on the use of MRI 
in GM are scarce, with a variety of definitions used for the description of MRI findings $(2,3,5,7,8,14,16)$. In suspected cases of GM, MRI should not lead to delayed biopsy and may be used as a complementary diagnostic method in addition to US and MG in order to exclude a diagnosis of inflammatory breast cancer in cases with symptoms of treatment-resistant mastitis (17). However, MRI can help in detecting lesions that cannot be visualized by US and MG due to parenchymal edema. In addition, since GM generally affects younger females, MRI may also be useful in cases where MG or US is inconclusive due to the character of the breast parenchyma. The most common MRI findings in our study were solitary or multiple separate or confluent abscesses of different sizes, with marked peripheral ring enhancement, accompanied by intensity changes suggesting edematous inflammation in the peripheral parenchyma, as well as non-mass-like heterogeneous segmental and regional contrast enhancement. In some cases, annular enhancing nodules, probably reflecting micro-abscesses, were only a few millimeters in size. These observations are similar to those reported by Kocaoglu et al. and Gautier et al. $(3,16)$. Previous studies have reported variable kinetic curve analysis results between different patients and different lesions $(2,5,16)$. In the study by Gautier et al., non-mass-like contrast-enhanced areas were generally associated with type 1 kinetic curves, while type 3 was the dominant kinetic curve in areas with annular contrast enhancement (3). In the present study, circumferential contrast enhancement was mostly associated with type 1 kinetic curves, while our findings were consistent with previous reports of non-mass-like contrast enhancement. In this study, MRI could more accurately and clearly identify the extent of disease and the typical findings of the inflammatory process, so it could more confidently classify lesions as BI-RADS 3, compared to conventional methods.

Several limitations of our study should be mentioned, including its retrospective and descriptive character. Second, the study group consisted only of patients with GM. Third, there were a limited number of patients who underwent MG and color Doppler US.

In conclusion, GM has no specific features on MG and US. MRI is a complementary diagnostic tool used with clinical and conventional radiological findings to assist in the diagnosis and differentiation of GM from malignant processes. A focal asymmetric density on MG associated with US findings of heterogeneous hypoechoic illdefined lesions and tubular extensions, along with MRI findings of ring-enhancing lesions associated with type 1 kinetic curves, can suggest the diagnosis of GM. However, histopathology remains essential for a definitive diagnosis and appropriate management.

\section{Footnotes}

Authors' Contributions: The study was designed by Pelin Seher Oztekin and Pinar Nercis Kosar. The data were collected by Pelin Seher Oztekin, Serap Erel, and Sema Hucumenoglu. Revision of the manuscript for intellectual content was performed by Pelin Seher Öztekin, Gamze Durhan, and Serap Erel.

Financial Disclosure: We affirm that we have no financial affiliations or involvement with any commercial organization, nor any financial interest related to the present manuscript

Funding/Support: The authors have no conflicts of interest with regard to this research or its funding.

\section{References}

1. Kessler E, Wolloch Y. Granulomatous mastitis: a lesion clinically simulating carcinoma. Am J Clin Pathol. 1972;58(6):642-6. [PubMed: 4674439].

2. Al-Khawari HA, Al-Manfouhi HA, Madda JP, Kovacs A, Sheikh M, Roberts O. Radiologic features of granulomatous mastitis. Breast J. 2011;17(6):645-50. doi: 10.1111/j.1524-4741.2011.01154.x. [PubMed: 21929558].

3. Gautier N, Lalonde L, Tran-Thanh D, El Khoury M, David J, Labelle $\mathrm{M}$, et al. Chronic granulomatous mastitis: Imaging, pathology and management. Eur J Radiol. 2013;82(4):165-75. doi: 10.1016/j.ejrad.2012.11.010. [PubMed: 23200627].

4. Heer R, Shrimankar J, Griffith CDM. Granulomatous mastitis can mimic breast cancer on clinical, radiological or cytological examination: a cautionary tale. The breast. 2003;12(4):283-6.

5. Ozturk M, Mavili E, Kahriman G, Akcan AC, Ozturk F. Granulomatous mastitis: radiological findings. Acta Radiol. 2007;48(2):150-5. doi: 10.1080/02841850601128975. [PubMed: 17354134].

6. Han BK, Choe YH, Park JM, Moon WK, Ko YH, Yang JH, et al. Granulomatous mastitis: mammographic and sonographic appearances. AJR Am J Roentgenol. 1999;173(2):317-20. doi: 10.2214/ajr.173.2.10430126. [PubMed: 10430126].

7. Boufettal H, Essodegui F, Noun M, Hermas S, Samouh N. Idiopathic granulomatous mastitis: a report of twenty cases. Diagn Interv Imaging. 2012;93(7-8):586-96. doi:10.1016/j.diii.2012.04.028. [PubMed: 22677299].

8. Yildiz S, Aralasmak A, Kadioglu H, Toprak H, Yetis H, Gucin Z, et al. Radiologic findings of idiopathic granulomatous mastitis. Med $\mathrm{Ul}$ trason. 2015;17(1):39-44. doi: 10.11152/mu.2013.2066.171.rfm. [PubMed: 25745656].

9. Canoy JM, Mitchell GS, Unold D, Miller V. A radiologic review of common breast disorders in pregnancy and the perinatal period. Semin Ultrasound CT MR. 2012;33(1):78-85. doi: 10.1053/j.sult.2011.10.002. [PubMed: 22264905].

10. Pistolese CA, Di Trapano R, Girardi V, Costanzo E, Di Poce I, Simonetti G. An unusual case of bilateral granulomatous mastitis. Case Rep Radiol. 2013;2013:694697. doi: 10.1155/2013/694697. [PubMed: 23781373].

11. Memis A, Bilgen I, Ustun EE, Ozdemir N, Erhan Y, Kapkac M. Granulomatous mastitis: imaging findings with histopathologic correlation. Clin Radiol. 2002;57(11):1001-6. [PubMed: 12409111].

12. Lee JH, Oh KK, Kim EK, Kwack KS, Jung WH, Lee HK. Radiologic and clinical features of idiopathic granulomatous lobular mastitis mimicking advanced breast cancer. Yonsei Med J. 2006;47(1):78-84. doi: 10.3349/ymj.2006.47.1.78. [PubMed: 16502488]. 
13. Hovanessian Larsen LJ, Peyvandi B, Klipfel N, Grant E, Iyengar G. Granulomatous lobular mastitis: imaging, diagnosis, and treatment. AJR Am J Roentgenol. 2009;193(2):574-81. doi: 10.2214/AJR.08.1528. [PubMed: 19620458].

14. Yaghan RJ. The magnetic resonance image findings of idiopathic granulomatous mastitis. Saudi Med J. 2004;25(11):1715-9. [PubMed: 15573210].

15. Sabate JM, Clotet M, Gomez A, De Las Heras P, Torrubia S, Salinas T. Radiologic evaluation of uncommon inflammatory and reactive breast disorders. Radiographics. 2005;25(2):411-24. doi: 10.1148/rg.252045077. [PubMed: 15798059].
16. Kocaoglu M, Somuncu I, Ors F, Bulakbasi N, Tayfun C, Ilkbahar S. Imaging findings in idiopathic granulomatous mastitis. A review with emphasis on magnetic resonance imaging. J Comput Assist Tomogr. 2004;28(5):635-41. [PubMed:15480037].

17. Engin G, Acunas G, Acunas B. Granulomatous mastitis: gray-scale and color Doppler sonographic findings. J Clin Ultrasound. 1999;27(3):1016. [PubMed: 10064406].

18. de Bazelaire C, Groheux D, Chapellier M, Sabatier F, Scemama A, Pluvinage A, et al. Breast inflammation: indications for MRI and PET-CT. Diagn Interv Imaging. 2012;93(2):104-15. doi: 10.1016/j.diii.2011.12.004. [PubMed: 22305594]. 